\title{
Indoor Intelligent Vehicle localization using WiFi Received Signal Strength Indicator
}

\author{
Nguyen Dinh-Van, Fawzi Nashashibi \\ RITS team \\ INRIA \\ Paris, France \\ Dinh-van.nguyen@inria.fr
}

\author{
Nguyen Thanh-Huong, Eric Castelli \\ PSI Dept., MICA Institute, CNRS - UMI 2954 \\ Hanoi, Vietnam \\ Thanh-huong.nguyen@mica.edu.vn,Eric.castelli@mica.edu
}

\begin{abstract}
Success of Intelligent Vehicle navigation largely depends on the ability to localize precisely the vehicle in the environment. In general, all intelligent vehicle seemed to agree on a combination of non-cumulative error localization method like GPS with more precise localization method but suffered from cumulative errors like Laser-SLAM with an odometer. However, as GPS is only available for outdoor environment and since the indoor environment is also an important scenario for intelligent vehicles, a replacement of GPS for indoor localization is required. Successfully replacing GPS will not only provide a reliable indoor localization method for vehicles but also keep the architecture of vehicle localizing system consistent and achieve a smooth transition from outdoor to indoor and vice versa. Often, movement speed for indoor vehicles will be as low as $10-12 \mathrm{~km} / \mathrm{h}$ [1] but still, it surpasses the movement speed of human walking $(3-5 \mathrm{~km} / \mathrm{h})$ and presents a challenge for a tight and complex environment. This paper proposes an improved WiFi-fingerprinting method to replace GPS behavior for the indoor environment. The key contribution is to use a raw data smoothing technique with an ensemble classification neural network method to deal with noisy WiFi signal strength. Also, environment constraints are applied to improve localization result. Experiments show this method is capable of replacing GPS for the indoor environment.
\end{abstract}

Keywords-Wifi signal, fingerprinting, neural network, classification, intelligent vehicle, low-speed, indoor localization.

\section{INTRODUCTION}

Research shows that $95 \%$ of time cars are in a parking area or an indoor environment[2]. It could be either car are maneuvering inside a parking area or parked completely there. But this is not a trivial problem. Studies in [1], [3] suggest an even more severe problem: for an average of $20-40$ minutes of searching and parking in Paris and Lyon, it costs France around 70 million hours of searching a year. Consequently, this leads to a 700 million euros loss not to mention others side-effect such as noise pollution, insecurity, air pollution and congestion. The final cost can be around a billion of euros wasted a year. An indoor navigation system for intelligent vehicles which allows autonomous parking can be a potential solution to this problem. (standard camera, depth camera, etc.) for indoor tracking of vehicles. These approaches achieve significant accuracy $(\sim 40$ $\mathrm{cm}$ at best case) but lack of coverage range (camera mounted in environment case) as well as subjecting to the lighting condition. Received frequency based approach is another method which is adopted widely. Studies in [8]-[10] use WiFi signal to determine the location of indoor robots/users in a known environment. A summary of indoor localization system can be found in TABLE I.

Still, these research has a common dilemma of movement speed constraint which is not likely to be specified. Within 3$5 \mathrm{~km} / \mathrm{h}$ of human walking speed, the localization behavior seems to comply with those stated in studies. As the movement of intelligent vehicles for an indoor area such as car park will be in the average of $10-12 \mathrm{~km} / \mathrm{h}$, it is necessary that another strategy is proposed. Also, as the outdoor localization architecture for vehicles is unified in a model of fusion of GPS and other sensors, the lack of GPS for the indoor environment require a sufficient replacement.

This paper proposes an improved work for indoor localization using WiFi Received Signal Strength Indicator (RSSI). The idea is to use a Fingerprinting approach with some raw data processing techniques, an ensemble classification with environment constraints to deliver a GPS behavior for the indoor environment.

TABLE I. SUMMARY OF INDOOR LOCALIZATION SYSTEM[11]

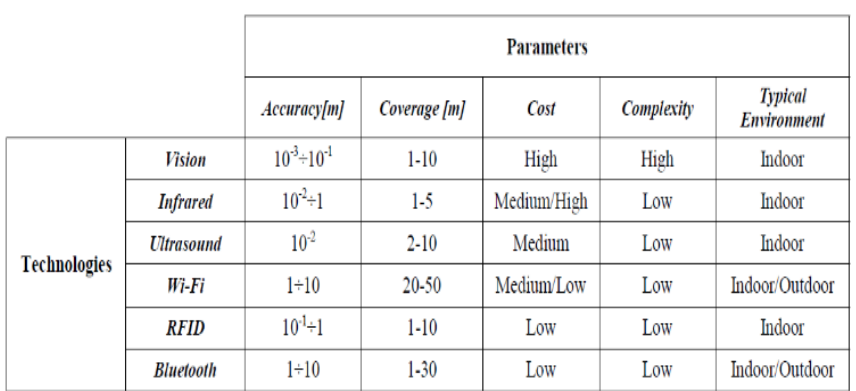

To deliver that goal, there are plenty of research for indoor localization of vehicles with a wide range of sensory approach. Vision techniques can be found in [4]-[7] utilize camera 


\section{Methodology}

\section{A. WiFi Fingerprinting localization}

WiFi fingerprinting localization is a solution based on learning the map of WiFi RSSI available in the environment at multiple reference points spread across the environment. The main assumption is each reference position with learned RSSIs of all available Access Points (APs) will be a unique feature which allows vehicles to recognize the location just by scanning RSSIs for next visit. This method has two steps. First is a training phase with multiples WiFi scanning at each reference position in an environment is recorded together with its coordinate. And second, a classification phase where new scanning of RSSIs without coordinate is compared to data recorded in step 1 to deliver a prediction of likely current position.

There are two major challenges in this approach: (1) RSSIs of standard WiFi system are often noisy due to interference and multipath propagation problem and (2) low scanning frequency of WiFi compare to the movement speed of vehicles. The first issue will be dealt with using some raw data processing technique, an ensemble classification method, and the second one will be addressed by applying environment constraints.

\section{B. Raw data processing}

Noisy and unstable of WiFi RSSI can be reduced by applying some raw data processing. Upon recording a vector of RSSI and the corresponding location as in (1) where $x_{i, j}$ is WiFi RSSI from $\mathrm{jth} \mathrm{WiFi}$ APs recorded in ith scan, $\rho_{l}$ is a label which has corresponding coordinate at position of sampling and $n$ is fixed constant (for learning algorithm purpose) which should be greater total number of APs in learning environment

$$
\left\{x_{i, 1}, x_{i, 2}, x_{i, 3}, \ldots, x_{i, n}, \rho_{l}\right\}
$$

Collected data will be normalized in the range of $[-1,1)$ where in particular scan, detected AP RSSI will be normalized (2) in the range $[0,1)$ with 0 as weakest possible signal strength and 1 as strongest possible signal strength. Other signals will be scored as undetected APs at $\rho_{l}$ and take value -1

$$
x_{i}=\left\{\begin{aligned}
-1, A P_{i} \text { undetected } \\
1-\frac{(-1) \times R S S I}{100}, A P_{i} \text { detected }
\end{aligned}\right.
$$

Also, to minimize the impact of interference, a system of 3 omnidirectional wifi antenna is mounted close to each other. In a particular scan, the antenna with highest RSSI will be recorded. The key observation here is interference and multipath propagation will only reduce received signal strength. Thus, higher RSSI will likely to be closer to direct signal without interference.

\section{Ensemble classification method}

A neural network is implemented to learn from training data and perform prediction in localization step. However, as RSSI appears to be noisy and scanning frequency of WiFi is low in comparison to movement speed, data will be considered to be a high variant. An algorithm is proposed using a method called Ensemble Bagging (Bootstrap Aggregating) Neural Network.
This method is well-known for combining multiple learning models to derive better results of prediction [12], [13].

Consider a classification method with a pair $\left\{X_{i}, Y_{j}\right\}$ where $X_{i}$ is a vector of predictor variable and $Y_{j}$ denotes a response, $Y_{j} \in\{1,2, \ldots m\}$. The target function is $P(Y=j \mid X=x)$ for classification. A function estimator which results from a set of training samples and a classification model is formed (3).

$$
\left.g(\cdot)=h\left(\left(X_{1}, Y_{1}\right),\left(X_{2}, Y_{2}\right), \ldots,\left(X_{n}, Y_{m}\right)\right)\right)
$$

Bagging algorithm consists following steps:

Step 1: Construct a bootstrap sample (4) by randomly sampling with replacement $n$ times from original data:

$$
\left(X_{1}^{\wedge}, Y_{1}^{\wedge}\right),\left(X_{2}^{\wedge}, Y_{2}^{\wedge}\right), \ldots,\left(X_{n}^{\wedge}, Y_{m}^{\wedge}\right)
$$

Step 2: Compute bootstrapped estimator $g^{\wedge}(\cdot)$ in (5) by applying same classification model to newly formed bootstrap sample.

$$
\left.g^{\wedge}(\cdot)=h\left(\left(X_{1}^{\wedge}, Y_{1}^{\wedge}\right),\left(X_{2}^{\wedge}, Y_{2}^{\wedge}\right), \ldots,\left(X_{\mathrm{n}}^{\wedge}, Y_{\mathrm{m}}^{\wedge}\right)\right)\right)
$$

Step 3: Repeat two steps above for $\mathrm{K}$ times with $\mathrm{K}$ is large. The bagging estimator is (6).

$$
g_{\text {bagg }}^{\wedge}(\cdot)=\frac{1}{K}\left(\sum_{i=1}^{K} g^{\wedge}(\cdot)\right)
$$

Theoretically, the bagging estimator is (7) as $\mathrm{K}$ goes to infinity:

$$
g_{\text {bagg }}^{\wedge}(\cdot)=\mathbf{E}^{\wedge}\left[g^{\wedge}(\cdot)\right]
$$

Thus, a finite large $\mathrm{K}$ in practice is expected to improve the accuracy of Monte Carlo approximation. In this study, a neural network is constructed and loosely tuned (weak learner). $\mathrm{K}$ is chosen at 50 for fifty simple neural networks.

\section{Environment constraints}

Since movement speed of vehicles will be around $2.5-3 \mathrm{~m} / \mathrm{s}$ and scanning frequency of $\mathrm{WiFi}$ receiver is as high as $1 \mathrm{~Hz}$. Thus it is necessary to include a simple motion model to interpolate possible vehicle positions since the last location. Due to small time different $(\Delta t=0.9 \mathrm{~s})$ for two consecutive scans, an average speed movement model is adopted here with speed profile is calculated as average speed for that particular duration.

Also, a simple environment with known obstacles and the movable path is formed using connection graph between reference positions. This information allows the system to remove jumping errors - where prediction results are jumping between adjacency reference points. Given the average speed movement model, a possible range of next location is calculated. As shown in Fig. 1 , with $\Delta t=0.9 \mathrm{~s}$, given a threshold $\varepsilon$, the next location should be within range of maximum reachable position with motion model (8).

$$
\left|d_{\text {travel }}-d_{\text {ref }}\right| \leq \varepsilon
$$




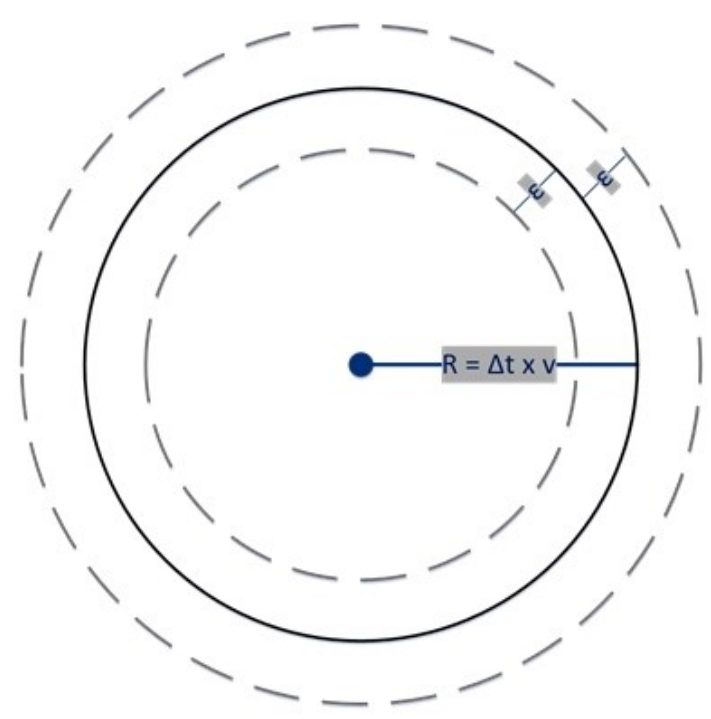

Fig. 1. Environment constraint with reachable positions

\section{EXPERIMENTS AND RESULTS}

\section{A. Experiments setup}

Experiments are conducted in campus site of INRIA Rocquencour, Paris. In order to achieve highly accurate ground truth, all experiments are performed outdoor, with an RTK (Real-Time Kinematic) GPS as a reference. This GPS allows us to have a $5-10 \mathrm{~cm}$ error of level 3 GPS. Experiment area is shown in Fig. 2 with 25 reference points each is $4 \mathrm{~m}$ away from each other.

The vehicle for experiments is a Cycab (Electric Intelligent Vehicle - studied by RITS team - INRIA) equipped with Odometer ( for speed profile), standard $2.4 \mathrm{GHz} \mathrm{WiFi}$ receiver and an RTK GPS antenna (Fig. 3). Maximum movement speed recorded is $2.5 \mathrm{~m} / \mathrm{s}$.

A site survey of average WiFi signal strength is studied across the testing path. The result shows in Fig. 4 is a heat map of WiFi signal strength with scale go from $[0,1)$ as 0 indicates $100 \mathrm{dbm}$ or not measured area and 1 indicates maximum possible signal strength. The conversion function is mentioned in section II.B.

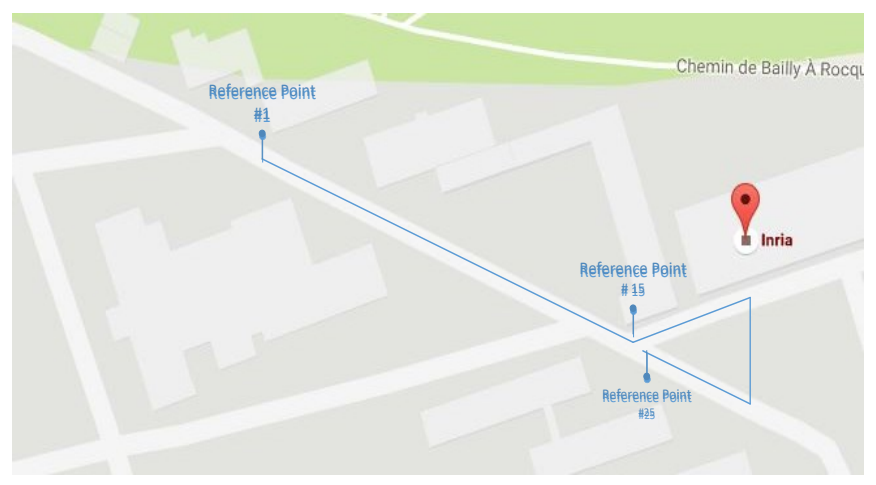

Fig. 2. Experiment sites with 25 reference points

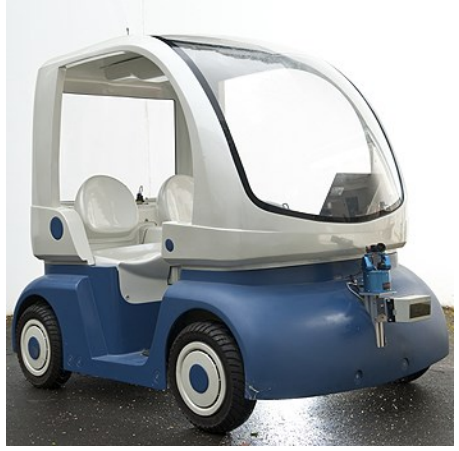

Fig. 3. Testing Vehicle Cycab - RITS team INRIA



Fig. 4. Site survey for average WiFi signal strength at Reference Path

Also, at each reference point, the number of Access Points detected is also studied to find a correlation between this factor and localization performance (Fig. 4). After a training phase of $30 \mathrm{WiFi}$ scans at each of 25 reference points, ten rounds of movement starting from reference point \#1 to reference point \#25 and go back to \#1 are tested. Fig. 5 shows 2-dimensions error of localization with red dots indicate $\mathrm{WiFi}$ prediction and green dots show real path recorded by RTK GPS.

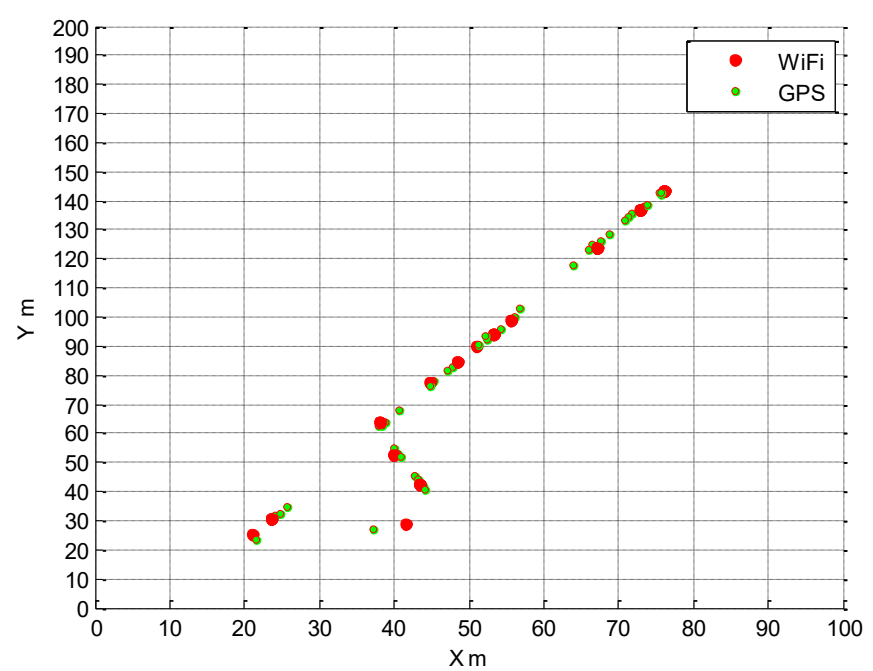

Fig. 5. Localization result in 2D 




Fig. 6. 3-Dimension movement path

TABLE II. SUMMRAY OF LOCALIZATION ERROR

\begin{tabular}{|l|l|l|l|}
\hline Total Distance & Maximum Erorr & Average Error & RMSQ Error \\
\hline $1012.9 \mathrm{~m}$ & $6.84 \mathrm{~m}$ & $2.25 \mathrm{~m}$ & $2.80 \mathrm{~m}$ \\
\hline
\end{tabular}

A 3-Dimension data with time synchronization shows in Fig. 7 also demonstrates the accuracy of WiFi localization. Summary of localization results show in TABLE II. with an average error of $2.25 \mathrm{~m}$ and Root Mean Square Error of $2.80 \mathrm{~m}$.

\section{CONCLUSION}

This paper presents an improved algorithm and technique for low-speed vehicles localization using WiFi Fingerprinting and Ensemble classification. The result of experiments shows a $2.25 \mathrm{~m}$ of average localization error. This result also indicates that the proposed method is capable of replacing standard GPS behavior for indoor intelligent vehicle navigation.

Although WiFi Fingerprinting alone is not sufficient for intelligent vehicles to move autonomously in an environment like parking area but together with other techniques such as Laser SLAM or cameras, it will not only offer a smooth transition from outdoor architecture to indoor architecture but also provide a cost-effective solution for indoor localization.

In the future, the study will focus on fusing this technique with Laser-SLAM for expected localization results of under $20 \mathrm{~cm}$.

\section{ACKNOWLEDGEMENT}

This project is funded by Hanoi University of Science and Technology under the grant number T2016-PC-104.

\section{REFERENCES}

[1] S. Belloche, "On-street Parking Search Time Modelling and Validation with Survey-based Data," Transp. Res. Procedia, vol. 6, no. June 2014 pp. 313-324, 2015.

[2] P. Barter, "CCars are parked 95\% of the time'. Let's check!," 2013 [Online]. Available: http://www.reinventingparking.org/2013/02/carsare-parked-95-of-time-lets-check.html. [Accessed: 24-Nov-2016].

[3] E. Gantelet and A. Lefauconnier, "The time looking for a parking space: Strategies, associated nuisances and stakes of parking management in France," pp. 1-7, 2006

[4] Q. Cai and J. K. Aggarwal, "Tracking Human Motion Using Multiple Cameras,” Pattern Recognit., pp. 68-72, 1996.

[5] I. Bouchrika, J. N. Carter, and M. S. Nixon, "Towards automated visual surveillance using gait for identity recognition and tracking across multiple non-intersecting cameras," Multimed. Tools Appl., pp. 12011221,2014

[6] R. Clark, N. Trigoni, and A. Markham, "Robust Vision-based Indoor Localization," Proc. 14th Int. Conf. Inf. Process. Sens. Networks, pp. 378 $379,2015$.

[7] P. Mirowski, R. Palaniappan, T. K. Ho, B. Labs, M. Avenue, and M. Hill, "Depth Camera SLAM on a Low-cost WiFi Mapping Robot," vol. 1, no. 908, pp. 0-5.

[8] F. Lemic, A. Behboodi, V. Handziski, and A. Wolisz, "Increasing Interference Robustness of WiFi Fingerprinting by Leveraging Spectrum Information," Comput. Inf. Technol. Ubiquitous Comput. Commun. Dependable, Auton. Secur. Comput. Pervasive Intell. Comput. (CIT/IUCC/DASC/PICOM), 2015 IEEE Int. Conf., pp. 1200 - 1208, 2015.

[9] J. Biswas and M. Veloso, "WiFi localization and navigation for autonomous indoor mobile robots," Proc. - IEEE Int. Conf. Robot. Autom., pp. 4379-4384, 2010.

[10] J. Huang, D. Millman, M. Quigley, D. Stavens, S. Thrun, and A Aggarwal, "Efficient, generalized indoor WiFi GraphSLAM," Proc. IEEE Int. Conf. Robot. Autom., pp. 1038-1043, 2011.

[11] L. Mainetti, L. Patrono, and I. Sergi, "A survey on indoor positioning systems," 2014 22nd Int. Conf. Software, Telecommun. Comput. Networks, pp. 111-120, 2014.

[12] T. G. Dietterich, "Ensem ble Methods in Mac hine Learning," Mult. Classif. Syst., vol. 1857, pp. 1-15, 2000.

[13] L. Breiman, "Bagging Predictors," Mach. Learn., vol. 24, pp. 123-140, 1996. 